\title{
Cervical Neuro-Muscular Syndrome: Discovery of a New Disease Group Caused by Abnormalities in the Cervical Muscles
}

\author{
Takayoshi MATSUI, ${ }^{1,2}$ Kunio II, ${ }^{1}$ Shuntaro HojO, ${ }^{2}$ and Keiji SANO ${ }^{2}$ \\ ${ }^{1}$ Japan Neurological Institute and Matsui Hospital, Kannonji, Kagawa; \\ ${ }^{2}$ Tokyo Neuro-Center, Tokyo
}

\begin{abstract}
Our previous study of whiplash injury found that abnormalities in the cervical muscles cause autonomic dystonia. Further research has found that abnormalities in the cervical muscles cause headache, chronic fatigue syndrome, vertigo, and dizziness. We named this group of diseases cervical neuro-muscular syndrome. Patients treated within a 2-year period from April 1, 2002 to March 31, 2004 reported good outcomes in $\mathbf{8 3 . 8 \%}$ for headache, $\mathbf{8 8 . 4 \%}$ for vertigo and dizziness, $\mathbf{8 4 . 5 \%}$ for chronic fatigue syndrome, $\mathbf{8 8 . 0} \%$ for autonomic dystonia, and $\mathbf{8 3 . 7 \%}$ for whiplash-associated disorder. A large number of outpatients present with general malaise, including many general physical complaints without identifiable cause. We propose that treatment of the cervical muscle is effective for general malaise.
\end{abstract}

Key words: whiplash-associated disorder, chronic fatigue syndrome, cervicogenic depression, tension type headache, vertigo

\section{Introduction}

The neck and lower back are two of the most injuryprone regions of the human body, and the neck is said to be even more fragile than the lower back. The spinal cord and the discs have been extensively investigated, but the cervical muscles have received little attention. Diseases caused by abnormalities in the external cervical muscles have barely been recognized. Almost all medical schools in Japan seem to be unaware of the clinical importance of cervical muscle functional anatomy, and only a few diseases involving the cervical muscles have been described. We believe that the cervical muscles are a significant blind spot in modern medicine.

Over the past 20 years, we have found evidence that abnormalities in the cervical muscles are responsible for many diseases that had once been thought to be idiopathic, or caused by other factors. We have also found that symptoms resolve after abnormalities in the cervical muscles disappear. Therefore, we propose that this group of diseases caused by the cervical muscles are part of a syndrome, named cervical neuro-muscular syndrome

Received September 14, 2010; Accepted October 25, 2011
(CNMS). The manifestations of CNMS may include chronic fatigue syndrome (CFS), headache, vertigo and dizziness, autonomous nerve dysfunction, and whiplash-associated disorder (WAD). We have emphasized conservative therapies over interventions as a method of treatment, because the causes and mechanisms of the diseases remain unclear. Both objective findings and subjective symptoms have disappeared after implementation of cervical muscle disease therapy.

Here we present some of our major findings regarding this novel syndrome, CNMS.

\section{Materials and Methods}

The diagnosis of CNMS is based on a focused interview of the medical history, palpation of the nuchal muscles, and radiography of the cervical vertebrae and intervertebral spaces, magnetic resonance (MR) imaging or computed tomography (CT) of the neck, and examination of the equilibrium, size, and movement of the pupils when exposed to light.

The pre-examination interview (consisting of 30 questions) is shown in Table 1 , and includes 23 questions originally presented in the 63rd Annual Meeting of the Japan Neurological Society in 2004. Patients who answered 'yes' to more than 10 of the 
Table 1 Pre-examination interview questions

1 Headache, or a sense of weight in head

2 Neck pain, or a sense of tension in neck

3 Stiff shoulders, or a sense of contracture in shoulder muscles

4 Prone to catch cold

5 Feel dizzy

6 Sense of unstableness while walking or standing

7 Feel like vomiting

8 Having trouble falling asleep

9 Unstable blood pressure

10 Difficulty staying in a warm environment

11 Profuse perspiration

12 Rapid heartbeat while at rest

13 Unclear and/or dim vision

14 Visual asthenia

15 Pain behind the eyes

16 Dry eye

17 Dry mouth

18 Low grade fever $\left(37-38^{\circ} \mathrm{C}\right.$, occasionally over $\left.38^{\circ} \mathrm{C}\right)$

19 Intestinal symptoms such as abdominal pain, constipation, and/or diarrhea

20 High demand for resting

21 Easy to tire

22 Loss of motivation

23 Symptoms worsen during bad weather, even on the day before

30 questions were considered to have moderate to severe abnormalities. Patients who answered 'yes' to more than 5 questions were considered to have mild abnormalities. Patients who answered 'yes' to fewer than 4 questions were considered to not require treatment, based on our experience of numerous previous cases. This classification was based on analysis of various complaints of patients with CNMS.

Palpation examines the musculus trapezius, musculus splenius capitis, musculus semispinalis capitis, musculus sternocleidomastoideus, musculus rectus capitis, musculus posterior major, and musculus obliquus capitis inferior. The 10 palpation points are shown in Fig. 1, as originally described at the Annual Meeting of the Japan Neurological Society in 1974. Muscle consistency and tenderness are examined in the upper, middle, and lower regions along the midline, between the external occipital protuberance and the prominent vertebra. Bilateral points are examined at the head of the nuchal posterior muscle group. Moreover, the consistency and tenderness of the musculus sternocleidomastoideus and the surrounding lateral cervical muscle group are examined.

Radiographs are taken using the anteroposterior, anterior flexion, neutral, and extension positions. Narrowing of intervertebral spaces is assessed. The arrangement of the seven cervical vertebrae is the most important consideration. The characteristics of the abnormal findings are shown in Fig. 2. The

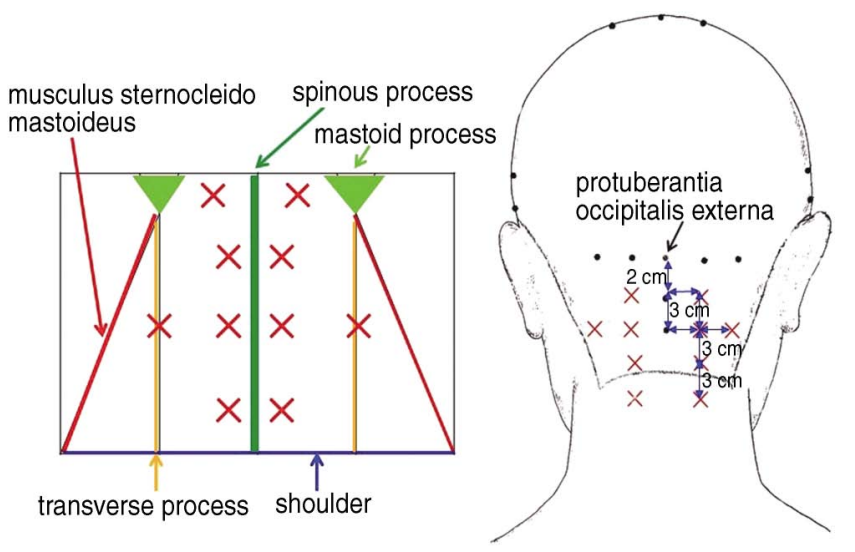

Fig. 1 Ten palpation points $(\times)$ and acupuncture points $(\bullet)$.
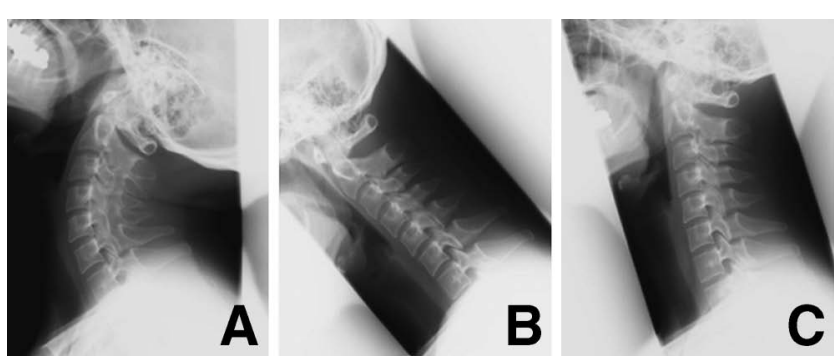

Fig. 2 Characteristic abnormal radiographic findings, in extension (A), in flexion (B), and in neutral position (C).

cervical vertebrae are found to lose normal curvature, and may become straight. The cervical vertebrae are not only curved abnormally, but also straight even in the anterior flexion position. Thus, loss of normal curvature and straight line deformity in the anterior flexion and/or neutral position are standard criteria for this syndrome. This characteristic was named 'straight sign' in 1978, and has since been considered as one of the main signs of CNMS.

MR imaging of the posterior cervical muscles reveals no abnormalities. However, MR imaging or CT do show protrusion of the cervical disc in the posterior direction. Our extensive experience suggests that patients with CNMS presenting with such disc protrusion to the posterior region causing compression takes about three times longer for recovery compared to patients without disc protrusion. Therefore, we recommend that MR imaging or CT is performed to check for protrusion of the C2-3, C3-4, C4-5, C5-6, C6-7, and C7-T1 discs in the posterior region.

Treatment of patients with CNMS depends on a 

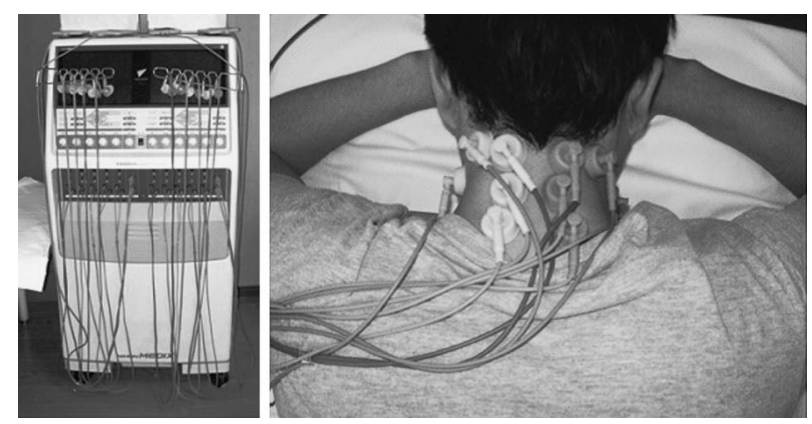

Fig. 3 Photographs showing low frequency therapy equipment.

combination of physical therapy, thermo therapy, oriental medicine, and pharmacological therapy. Muscular abnormalities detected by palpation of the 10 check points are treated by low current frequency therapy (Fig. 3). Acupuncture therapy was administered to the specified 10 points (Fig. 1), which are different from the points typically addressed in classical acupuncture. Depolarizing electric conduction was administered using needles. Far-infrared radiation was applied to the 10 points for low current frequency therapy.

\section{Results}

Table 2 shows the results of therapy for CNMS over the 2-year period from April 1, 2002 to March 31, 2004, in which all examinations were done uniformly. We found that many symptoms disappeared after treatment of the cervical muscle. Patients with headache reported permanent relief in 62 of 74 cases $(83.8 \%)$. Even physicians at an outpatient clinic specialized in a headache only expect to temporarily ease headache using medication. However, we found that tension type headache recovered completely in a high number of cases after treating the cervical muscle. Patients with vertigo and dizziness reported resolution in 76 of 86 cases (88.4\%), including all types of dizziness. Few physicians understand the extent of vertigo and dizziness caused by cervical muscles. Patients with autonomic dystonia (imbalance) reported resolution in 81 of 92 cases (88\%). Patients with WAD reported healing in 41 of 49 cases $(83.7 \%)$. Patients with CFS reported improvement in 22 of 26 cases (84.5\%). These results far exceed the outcomes of conventional treatment.

Illustrative Case 1: A 48-year-old man with WAD was involved in a rear-end traffic collision, and suffered headache, nuchal pain, shoulder stiffness, and dizziness. He started to experience sudden on-
Table 2 Outcomes of the therapy

\begin{tabular}{llc}
\hline \multicolumn{1}{c}{ Symptom } & Outcome & No. of cases \\
\hline Headache & healed & $62 / 74(83.8 \%)$ \\
& improved & $10 / 74(13.5 \%)$ \\
Unchanged & $2 / 74(2.7 \%)$ \\
Vertigo, dizziness & healed & $76 / 86(88.4 \%)$ \\
& improved & $7 / 86(8.1 \%)$ \\
Autonomic dystonia & unchanged & $3 / 86(3.5 \%)$ \\
& healed & $81 / 92(88.0 \%)$ \\
Whiplash injury & unchanged & $11 / 92(12.0 \%)$ \\
& healed & $41 / 49(0 \%)$ \\
& improved & $8 / 49(16.3 \%)$ \\
Chronic fatigue syndrome & unchanged & $0 / 49(0 \%)$ \\
& healed & $22 / 26(84.5 \%)$ \\
& improved & $4 / 26(15.5 \%)$ \\
& unchanged & $0 / 26(0 \%)$ \\
\hline
\end{tabular}

set of palpitation tachycardia, heat in his head, and hypothermia in the lower half of his body. He had to change his underwear three times a day due to hyperhidrosis. He could not keep his eyes open in bright environments due to excessive light intensity. He had to lie down throughout the day because of general fatigue and lethargic state. He consulted several cardiologists for palpitations but no abnormalities were detected, and the cause remained unknown. He visited various physicians but the symptoms did not improve. On admission to our hospital, 16 of 23 interview questions were found to be positive (Fig. 4A, B). Two weeks after physical therapy and medication, his symptoms were reduced. After the therapy, the objective findings of the cervical muscles also improved, and both objective findings and symptoms disappeared 7 weeks later.

Illustrative Case 2: A 37-year-old man with CFS lost enthusiasm for everything, had general fatigue, and became unable to work without any obvious cause. He had a sense of heat in the nuchal region and felt slight to moderate fever. He consulted more than 10 physicians and underwent various examinations to identify causes without success. On his first visit to our clinic, his cervical muscles were found to be more consistent and tender at all 10 nuchal check points (Fig. 4C, D). A total of 18 of the 23 interview questions were positive. On admission to the hospital, he underwent pharmacological and physical therapies to treat his posterior cervical muscles, resulting in marked improvement of symptoms after 2 weeks. One month later, almost all symptoms had disappeared together with abnormal findings in the posterior cervical muscles. Since discharge from our 

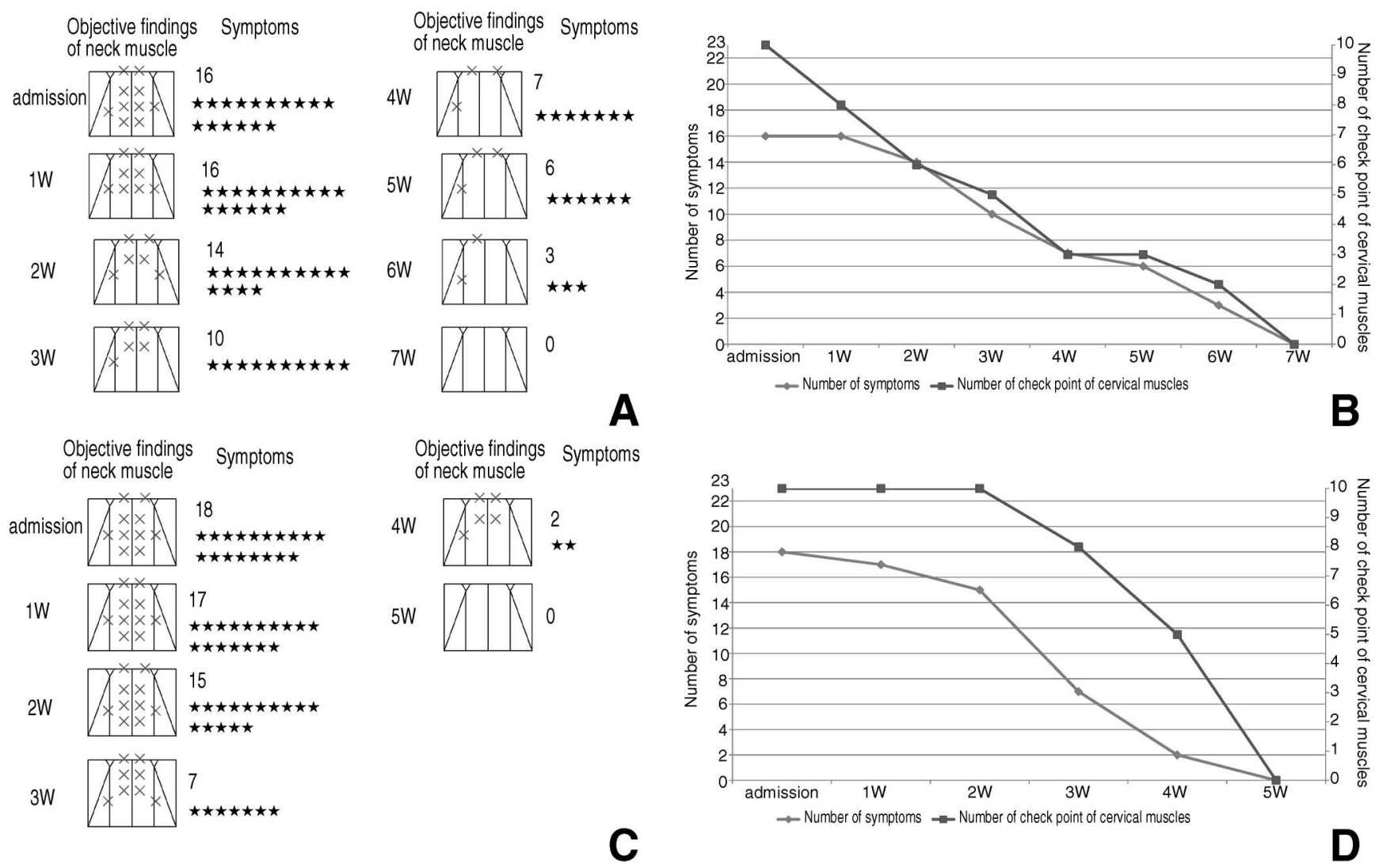

Fig. 4 Diagrams and graphs showing the location and number of abnormalities in the cervical muscles, and the number of symptoms. A, B: Case 1 with whiplash-associated disorder. C, D: Case 2 with chronic fatigue syndrome. W: week(s).

hospital, he has been working normally and remained healthy. No recurrence has occurred during 2 years of follow-up observation.

\section{Discussion}

Autonomic nervous system abnormality is caused by imbalance of the sympathetic and the parasympathetic nervous systems, that is an activated sympathetic nervous system and a suppressed parasympathetic nervous system. Almost all patients with CNMS show characteristically dilated pupils when exposed to light. Frequently reported symptoms include blurred vision, asthenopia, dazzling (patient cannot keep the eyes open), and ophthalmic pain. Weakness in pupil contraction is also found in the light reaction test. Our past studies have shown that patients with severe abnormalities of the posterior cervical muscles show almost no contraction or rapid dilation of the pupils, particularly if their pupils are large. Pupils normally show some contraction when exposed to light. Contracted pupils dilate rapidly in patients with severe CNMS. Hippus phenomenon is often seen in subjects with a good balance of sympathetic and parasympathetic nerve functions. Abnormalities of the posterior cervical muscles and the number of symptoms are correlated, i.e., the number of symptoms decreases as abnormalities in the posterior cervical muscles improve. Objective findings always improve with resolution of subjective symptoms. CNMS symptoms and many other symptoms such as "psychogenic reaction," panic syndrome, and stress syndrome can be treated with our combined therapy.

Increasing numbers of people are suffering from CFS nowadays, as often reported in newspapers. As a matter of fact, some researchers estimate that there are about four million CFS patients in the USA. CFS patients show various symptoms, such as sore throat and slight fever, suggesting the possibility of viral infection. Some studies report that the virus may be important in the CFS. CNMS patients claim generalized fatigue, slight fever, and loss of motivation. Many patients become unable to go to school or work.

Our past studies have found that patients develop 
infections in the upper respiratory tract due to irregular body temperature control. Some CNMS patients have no overt fever, but only slight fever. However, the cause of such slight fever has not been identified. Various CNMS symptoms including slight fever, and objective findings in the posterior cervical muscles, decrease after administration of CNMS therapy. However, the mechanism of the fever remains unknown.

Most CNMS patients complain of general fatigue and loss of motivation. Administration of our therapy improved the symptoms in more than $80 \%$ of patients with general fatigue and loss of motivation. Symptoms of CFS improved in a considerable number of patients after CNMS treatment. Despite extensive research, no viruses have been identified as a causal factor of CFS. One of the main arguments for viral infection is the occurrence of multiple cases of CFS in a restricted area.

Since many people currently suffer from CFS, it is easy to assume that multiple occurrences of patients with CFS can occur in areas of low atmospheric pressure, which appears to be a region with restricted infection. Our previous studies have found that the occurrence of CNMS correlates with low atmospheric pressure. Therefore, we suggest that CFS may be one of the symptoms of CNMS.

Further study is required to establish dizziness as a CNMS symptom. Most patients diagnosed with Meniere syndrome at other hospitals improved after administration of our CNMS therapy. Some patients with suspected Meniere syndrome associated with strong vertigo, dizziness, and vomiting may be cured by CNMS therapy. No recurrences have been observed in some patients with repeated paroxysms of Meniere syndrome for many years. Therefore, CNMS treatment was effective in treating Meniere syndrome in most cases. Dizziness was more frequently observed than vertigo in patients with CNMS. Vertigo can also be improved by treating cervical muscle abnormalities.

Headache $^{4)}$ can be classified into various types. About $95 \%$ of patients who underwent treatment at the outpatient center of our hospital had a cervical origin of headache. Medication is needed for patients with vascular headaches, which are caused by dilation of blood vessels and migraine. With the exception of these patients, most outpatients had abnormalities in the posterior cervical muscles, which were later resolved by the CNMS therapy. No additional therapy was required. However, CNMS therapy was not effective in patients with severe cervical spondylosis and protrusion of the cervical disc.

No definitive conclusions have been established regarding the mechanisms of therapies for WAD for the last 20 years. In our investigations of CNMS, we found patients with WAD had abnormalities in the posterior cervical muscles. Our experience shows that $83.7 \%$ of patients were cured, and $100 \%$ were improved, as a result of therapy administered after determination of the type of abnormality, and of the specific location of the abnormalities in the posterior cervical muscles.

We can also now better detect malingering patients who claim pseudo syndromes, or pretend to be seriously injured to obtain compensation, in the absence of objective findings possessed by the patient. WAD has been assumed to result from abnormalities in the cervical soft tissues. Many symptoms disappeared after intensive administration of CNMS treatment to abnormal regions of the posterior cervical muscles. In other words, symptoms disappeared in parallel with the improvement of objective findings.

Headache, thoracic pain, ill health, deep disturbance, stiff aches, fatigue, and depression have all been described as symptoms of WAD. ${ }^{2)}$ These symptoms are also considered to be the main symptoms of CNMS. However, "too many things remain unknown and this common syndrome is wrapped in a shroud of mystery." 7 ) Many researchers have reported similar ideas. ${ }^{3,5,6)}$ We have determined that WAD is caused by abnormalities in the posterior cervical muscles and that the symptoms disappear in parallel with improvement of objective findings in these muscles after CNMS treatment is administered. Barré-Lieou syndrome has been well known since 1925 when Barré reported that excruciating extension and flexion stimulate the sympathetic nervous system of the posterior cervical muscle region to cause headache, a heavy sense in the head, facial pain, dizziness, facial redness, and pharyngeal sensory abnormality. ${ }^{1)}$

However, we observed that headache is caused by compression of the nervus occipitalis major due to increased consistency and muscle tone of the musculus semispinalis, which compresses the nervus occipitalis, and that the headache is reduced and then disappears completely with treatment of the posterior cervical muscles. Compression of the cervical muscle does not cause excitement but rather dysfunction of the autonomic nerves. Specifically, we speculate that various autonomic nervous symptoms are provoked by hypofunction of the parasympathetic nerves. Clinically, various autonomic nerve symptoms decrease and disappear in parallel with decreased consistency and muscle tone of the cervical muscles.

Our present study has clarified the method of treating various symptoms caused by autonomic 
nerve dysfunction, headache and dizziness. Based on previous studies, we speculate that dysfunction of the parasympathetic nerves cause Barré-Lieou syndrome, which has been thought to be caused by excitement of sympathetic nerves. Many patients showed not only mydriasis but also no reaction to light for many years, which indicates dysfunction of the parasympathetic nerves. Since miosis in reaction to light does not occur without parasympathetic nerve action, mydriasis with posterior cervical muscular abnormalities suggests dysfunction of the parasympathetic nerves.

The present findings indicate that disorders of the cervical muscle are a medical blind spot. Abnormality of the cervical muscle was often associated with tension type headache, vertigo and dizziness, autonomic dystonia, WAD, CFS, etc., and more than $80 \%$ of patients with these diseases were cured by treatment of the cervical muscle. We propose that diseases caused by cervical muscle abnormalities form a syndrome, CNMS.

\section{References}

1) Barré MJ: Sur un syndrome sympathique cervical postérieur et sa cause fréquente, l'artrite cervicale. Rev Neurol (Paris) 1: 1246-1248, 1926 (French)
2) Berglund A, Alfredsson L, Jensen I, Cassidy JD, Nygren A: The association between exposure to a rear-end collision and future health complaints. J Clin Epidemiol 54: 851-856, 2001

3) Eck JC, Hodges SD, Humphreys SC: Whiplash: a review of a commonly misunderstood injury. Am J Med 110: 651-656, 2001

4) Gesztelyi G, Bereczki D: Determinants of disability in everyday activities differ in primary and cervicogenic headaches and in low back pain. Psychiatry Clin Neurosci 60: 271-276, 2006

5) Gracovetsky S, Newman N, Pawlowsky M, Lanzo V, Davey B, Robinson L: A database for estimating normal spinal motion derived from noninvasive measurements. Spine (Phila Pa 1976) 20: 1036-1046, 1995

6) Kasch H, Stengaard-Pedersen K, Arendt-Nielsen L, Staehelin Jensen T: Headache, neck pain, and neck mobility after acute whiplash injury: a prospective study. Spine (Phila Pa 1976) 26: 1246-1251, 2001

7) Rosenfeld RPT M: Whiplash. Am J Med 110: 667-668, 2001

Address reprint requests to: Takayoshi Matsui, MD, Japan Neurological Institute and Matsui Hospital, 739 Muraguro-cho, Kannonji, Kagawa 768-0013, Japan. e-mail: t.matsui@matsui-hp.com 